\title{
Characteristics of Neonatal Hyperbilirubinemia at West Java's Top Referral Hospital, Indonesia
}

\author{
Nurain Yahya, ${ }^{1}$ Tetty Yuniati, ${ }^{2}$ Leonardo Lubis ${ }^{3}$ \\ ${ }^{1}$ Faculty of Medicine Universitas Padjadjaran, ${ }^{2}$ Department of Child Health Faculty of Medicine \\ Universitas Padjadjaran/Dr. Hasan Sadikin General Hospital Bandung, ${ }^{3}$ Department of Anatomy, \\ Cell Biology and Physiology Faculty of Medicine Universitas Padjadjaran
}

\begin{abstract}
Background: Neonatal hyperbilirubinemia or neonatal jaundice is a common disorder and considered as one of the main problems in neonatal period worldwide with high incidence, especially in Asia and Southeast Asia region. This study was conducted to determine the characteristics of neonatal hyperbilirubinemia including the prevalence and frequency of neonatal hyperbilirubinemia causes at Dr. Hasan Sadikin General Hospital in 2012.

Methods: This descriptive study was conducted at Dr. Hasan Sadikin General Hospital using the crosssectional method. Four hundred and twenty five cases of neonatal hyperbilirubinemia in hospitalized and outpatients, from 1 January to 31 December 2012 were included in this descriptive study.

Results: The prevalence of neonatal hyperbilirubinemia was about $4.08 \%$. Causes were identified in 95 cases and the most common cause of neonatal hyperbilirubinemia during this period of time was physiological causes $(23.2 \%)$. Other causes included neonatal hepatitis $(14.7 \%)$, other hemolytic causes (13.7\%), infection (12.6\%), ABO incompatibility (11.6\%) and breastfeeding $(11.6 \%)$.

Conclusions: Neonatal hyperbilirubinemia cases are common and remain as one of the neonatal problems in Dr. Hasan Sadikin General Hospital, thus prevention and proper management of neonatal hyperbilirubinemia should be implemented.
\end{abstract}

Keywords: Neonatal hyperbilirubinemia, neonatal jaundice, prevalence

\section{Introduction}

Neonatal hyperbilirubinemia or neonatal jaundice is a condition cause by an elevation of the serum bilirubin level. ${ }^{1-3}$ It is common and considered as one of the main problems in neonatal period worldwide with high incidence, especially in Asia and Southeast Asia region. ${ }^{3,4}$

In the course of this disorder, severe neonatal hyperbilirubinemia can cause acute bilirubin encephalopathy, with deafness and other permanent neurodevelopmental abnormalities due to brain toxicity of bilirubin occurrence. ${ }^{5,6}$

Neonatal hyperbilirubinemia can be classified into physiologic jaundice and pathologic cause of jaundice. ${ }^{7}$ Determination of pathologic causes is pursued when the time and course of jaundice are significantly different from that of physiologic jaundice, by using laboratory tests. ${ }^{3}$

The number of neonatal hyperbilirubinemia cases is much lower in developed countries than in the developing countries. ${ }^{7,8}$ There were a few studies conducted in Indonesia recently to determine the prevalence of neonatal hyperbilirubinemia, as well as the frequency of causes. Data from the Perinatalogy Ward at Dr. Hasan Sadikin General Hospital ${ }^{9}$ reported the prevalence of neonatal hyperbilirubinemia among full term neonates in 2008 was approximately $17.53 \%$. Another study conducted at Dr. Soetomo General Hospital ${ }^{10}$ in 2000 , showed that the prevalence of neonatal hyperbilirubinemia was $13 \%$.

Studies about determining the frequency of causes of neonatal hyperbilirubinemia were not extensive due to resource limitations and reported majority of the causes were

Correspondence: Nurain Yahya, Faculty of Medicine, Universitas Padjadjaran, Jalan Raya Bandung-Sumedang Km.21, Jatinangor, Sumedang, Indonesia, Phone: +62 85722143865 Email: escape_k@yahoo.com 
unidentified. ${ }^{11}$ This study was conducted to determine the characteristics of neonatal hyperbilirubinemia including the prevalence and the frequency of causes of neonatal hyperbilirubinemia at Dr. Hasan Sadikin General Hospital in 2012.

\section{Methods}

The study was conducted at Dr. Hasan Sadikin General Hospital, using the crosssectional method with purposive sampling. The subjects were enlisted from hospitalized neonatal hyperbilirubinemia patients and the neonates born from 1 January 2012 to 31 December 2012 at Dr. Hasan Sadikin General Hospital. A total of 425 neonates diagnosed with neonatal hyperbilirubinemia were involved in this descriptive study. Neonatal hyperbilirubinemia refers to the presence of yellowish discoloration of the skin and sclera, associated with an increase level of serum bilirubin. ${ }^{3}$ Some of the babies were excluded if they had multiple congenital abnormalities.

The prevalence was calculated by using the prevalence formula. The total number of neonatal hyperbilirubinemia cases was obtained from hospitalized patients and newborns. The denominator of the prevalence formula included the number of live births and the total number of pediatric patients who came to the pediatric clin in 2012. The list of identified causes of neonatal hyperbilirubinemia from these patients was collected from medical records and the frequency of causes was determined. The characteristics of the subject population were summarized using descriptive statistics. Approval from the Health Research Ethics Committee was acquired before the collection of data.

\section{Table 1 Characteristics of Study Population}

\begin{tabular}{lcc}
\hline \multicolumn{1}{c}{ Characteristics } & Number of Patients* (n=425) & Percentage (\%) \\
\hline Sex & & \\
Male & 250 & 58.8 \\
Female & 175 & 41.2 \\
Birth & & \\
$\quad$ Term infant ( $\geq 37$ weeks) & 303 & 71.3 \\
$\quad$ Preterm infant (<37 weeks) & 122 & 28.7 \\
Type of delivery & & \\
Caesarean section & 245 & 57.6 \\
Spontaneous & 156 & 36.7 \\
Vacuum extraction & 13 & 3.1 \\
Forceps delivery & 11 & 2.6 \\
Birth weight, Low birth weight $(<2500)$ & 159 & 37.4 \\
$\quad$ Normal birth weight $(\geq 2500)$ & 266 & 62.6 \\
Size for gestational age & & \\
Appropriate for gestational age & 368 & 86.6 \\
Small for gestational age & 42 & 9.9 \\
Large for gestational age & 15 & 3.5 \\
Death & & \\
Less than 48 hours & 2 & 0.5 \\
More than 48 hours & 10 & 2.4 \\
No & 413 & 97.2 \\
\hline
\end{tabular}

Note: SD = Standard deviation

* Unless stated otherwise 
Table 2 Distribution of Neonatal Hyperbilirubinemia Patients

\begin{tabular}{lcc}
\hline Neonatal Hyperbilirubinemia Patients & Frequency (n) & Percentage (\%) \\
\hline Inpatients & 171 & 40.24 \\
Newborns & 254 & 59.76 \\
Total & 425 & 100.00 \\
\hline
\end{tabular}

\section{Results}

The study was conducted at Dr. Hasan Sadikin General Hospital, and data was collected from medical records of patients diagnosed with neonatal hyperbilirubinemia in the period between 1 January and 31 December 2012.

A higher number of male patients constituted the total number of neonatal hyperbilirubinemia cases. Most of the infants were term infants and had appropriate size for gestational age. Those delivered through Caesarean section constituted a higher proportion of the total number of neonatal hyperbilirubinemia patients, compared to those through other types of vaginal deliveries (Table 1).

The prevalence of neonatal hyperbilirubinemia at Dr. Hasan Sadikin General Hospital in 2012 was about 4.08\%. Hospitalized neonatal hyperbilirubinemia patients contributed greater proportion of the total number of neonatal hyperbilirubinemia patients than neonatal hyperbilirubinemia patients among neonates born at Dr. Hasan Sadikin General Hospital (Table 2).

Furthermore, several causes for neonatal hyperbilirubinemia were obtained from medical records in 95 cases $(22.4 \%)$. However, no cause was identified in the remaining cases $(77.6 \%)$, which represented the majority. The frequency of identified causes leading to presence of neonatal hyperbilirubinemia conditions showed that the main cause of neonatal hyperbilirubinemia was due to physiological causes. The second most common cause was neonatal hepatitis, followed by other hemolytic causes and infection (Figure 1).

Patients with complications of severe neonatal hyperbilirubinemia included 4 neonates with bilirubin encephalopathy and a neonate with kernicterus (chronic bilirubin encephalopathy). However, only the kernicterus patient died before 48 hours after birth with neonatal sepsis as the cause

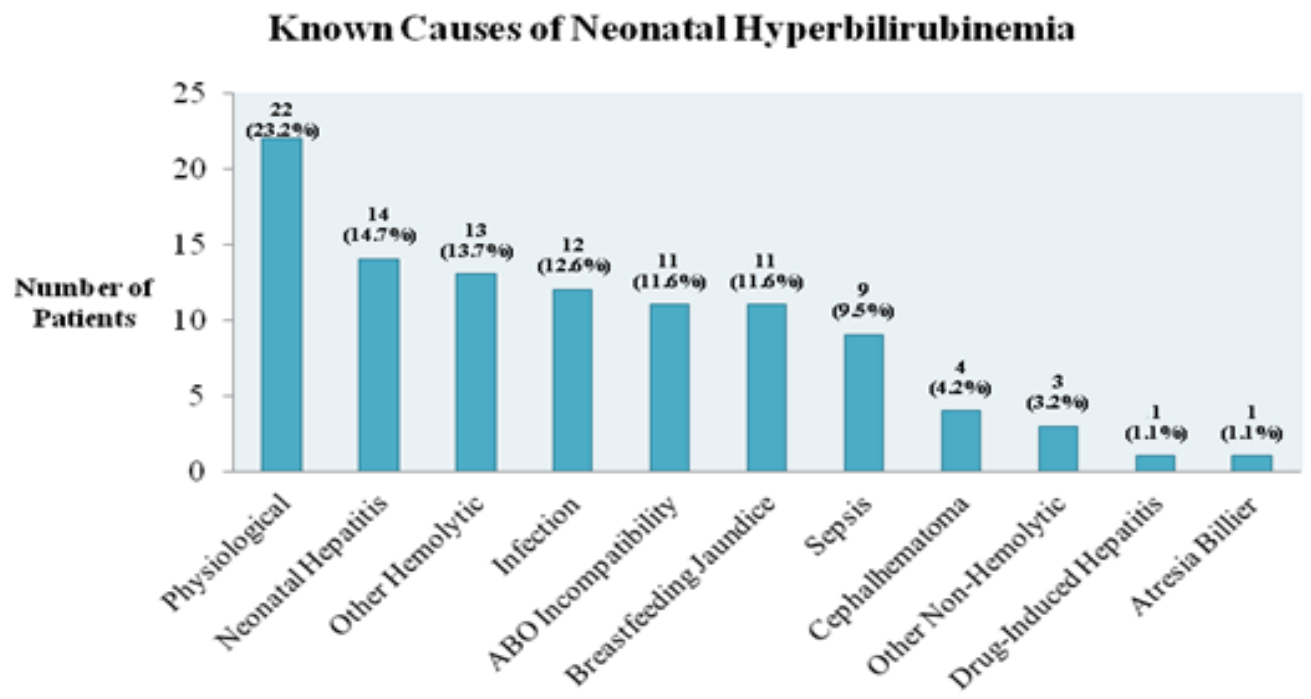

Figure 1 Frequency and Causes of Neonatal Hyperbilirubinemia 
Table 3 Feeding History of Hospitalized Neonatal Hyperbilirubinemia Patients

\begin{tabular}{lcc}
\hline \multicolumn{1}{c}{ Feeding Type } & Frequency $(\mathbf{n = 1 7 1})$ & Percentage (\%) \\
\hline Exclusive breastfeeding & 135 & 78.9 \\
Mixed & 31 & 18.1 \\
Formula milk & 5 & 2.9 \\
\hline
\end{tabular}

of death. Additionally, 12 neonates died and the causes of death were mainly sepsis and neonatal hepatitis.

Moreover, according to history acquired from mothers of 171 hospitalized patients reported information related with the risk factors of neonatal hyperbilirubinemia such as any maternal illness during pregnancy, any previous sibling with jaundice and breastfeeding. Exclusive breastfeeding was the most favorable diet given by the mothers of neonatal hyperbilirubinemia infants, followed by mixed feeding (breastfeeding and formula milk) and formula milk (Table 3).

Four hospitalized patients had history of the first sibling with jaundice. However, no cause was known and no further information if any sibling required phototherapy for neonatal jaundice. The number of cases was 3, 4 and 1 related cases in mother with history of infection or illness during pregnancy, hypertension and diabetes mellitus, respectively. Only 7 mothers aged older than 25 years were reported among the hospitalized patients.

Re-admission of discharged patients occurred with a chief complaint of jaundice accounted for 7 patients with mean peak total bilirubin of $15.68 \mathrm{mg} / \mathrm{dl}$ (Standard deviation $5.70 \mathrm{mg} / \mathrm{dl}$ ). All these patients received phototherapy as the treatment. A total of 137 neonates from hospitalized neonatal hyperbilirubinemia received phototherapy and of these, only 4 neonates needed to undergo exchange transfusion. There were 5 neonates from hospitalized neonatal hyperbilirubinemia who required exchange transfusion.

\section{Discussions}

The study showed that the prevalence of neonatal hyperbilirubinemia at Dr. Hasan Sadikin General Hospital in 2012 was 4.08\%. The prevalence was lower than the prevalence of neonatal hyperbilirubinemia in the same location in 2008, which was reported as $17.53 \%{ }^{9}$ The previous study included full term infants as the subject population, while in this study both full term and preterm infants were included. Descending prevalence may be due to many neonatal hyperbilirubin patients were actually managed by other health workers before coming to hospital, as the more severe conditions of neonatal hyperbilirubinemia were referred to the hospital, thus reducing the number of neonatal hyperbilirubinemia at Dr. Hasan Sadikin General Hospital. Otherwise, it could be a sign assuming that proper prevention of neonatal hyperbilirubinemia guidelines published by American Academy of Pediatrics (AAP) was implemented and improved. ${ }^{12}$

It is essential for physicians to distinguish whether the jaundice is physiologic or pathologic. Benign physiologic jaundice is caused by increased production of bilirubin and immature liver function for bilirubin clearance. $^{3}$ Increased bilirubin production is caused by increased of ineffective erythropoiesis, increased of volume and decreased survival of red blood cells in infants. ${ }^{13}$ Undetermined causes of neonatal hyperbilirubinemia constituted majority of cases in this study was similar to studies conducted in Canada $^{8}$ and Pakistan ${ }^{11}$. Many cases without reported causes in the medical records contributed to limitations in this study. Economical constraints caused many patients were unable to afford to undergo several diagnostic tests; the patients' family had to ask for early discharge from the hospital without determining the cause of neonatal hyperbilirubinemia.

Early onset of jaundice (less than 24 hours) is pathologic and can be caused by infection, spherocytosis, or hemolytic disease. Hemolytic diseases due to ABO incompatibility and glucose-6-phosphate dehydrogenase (G6PD) deficiency lead to overproduction of bilirubin. ${ }^{13}$ Eleven cases of neonatal hyperbilirubinemia with $\mathrm{ABO}$ incompatibility were reported in the study, compared to a study conducted in Canada $^{8}$ which presented $\mathrm{ABO}$ incompatibility as the most common cause of severe neonatal hyperbilirubinemia, followed by G6PD deficiency. However, no G6PD deficiency cause was found in this study because G6PD enzyme test was not performed, perhaps due 
to economical and resource constraints.

Other pathologic causes such as neonatal hepatitis, infections, biliary atresia and sepsis were identified from the cases in this study. Neonatal hepatitis was the second most common cause and the examples of the etiologies reported were cytomegalovirus (CMV) and toxoplasmosis, other (syphilis, varicella-zoster, parvovirus B19), rubella, cytomegalovirus, and herpes (TORCH) infections. Additionally, sepsis condition leads to mixed overproduction and under-secretion of bilirubin. ${ }^{13}$ Biliary atresia was reported in one case diagnosed with cholestasis jaundice causing presentation of hyperbilirubinemia through mechanism of biliary obstruction leading to unsecretion of bilirubin. ${ }^{13}$

Moreover, risk factors for development of severe hyperbilirubinemia included male sex, mothers older than 25 years, poorly established exclusive breastfeeding, sibling had jaundice and any sibling required phototherapy for neonatal jaundice. ${ }^{12}$ The higher number of male patients than female patients in this study corresponded with one of the risk factors. Mothers older than 25 years of age have higher risk of pregnancy complications, such as hypertension and diabetes. ${ }^{14}$ Infants of diabetic mothers are at risk of hyperbilirubinemia due to larger red cell mass, inefficient conjugation by the relative immature hepatic enzyme system and ineffective erythropoiesis, leading to increased serum level of unconjugated bilirubin. $^{15}$ Furthermore, breastfeeding jaundice was reported in 11 patients. These patients presented with mild dehydration and the mother mentioned that their babies did not want or were lazy to be breastfed. Breastfeeding jaundice may occur due to inadequate intake and ineffective breastfeeding, thus resulting in increased enterohepatic circulation and delayed bilirubin elimination. ${ }^{13}$

One of the major risk factor for developing severe hyperbilirubinemia is cephalohematoma or any significant bruising. ${ }^{12}$ In this study, 4 neonates had cephalohematoma, which led to overproduction of bilirubin due to breakdown of extravascular blood. ${ }^{13}$ Besides cephalohematoma can be caused by laceration during delivery due to the use of vacuum extraction, and traumatic injury in macrosomia babies. ${ }^{16}$

Furthermore, a kernicterus (chronic bilirubin encephalopathy) case was found in this study; even it is rare since the implementation of phototherapy and exchange transfusion. ${ }^{6,17,18}$ The patient presented with apnea, which was the late effect of bilirubin toxicity. However, both the developed and developing countries are still reporting the occurrence of kernicterus, which can be prevented through proper identification and management of progression of severe neonatal hyperbilirubinemia. ${ }^{6,18,19}$

There were several limitations in this study. A number of medical records were missing and could not be tracked. More efficient administration process of getting approval letter and proper management of the medical records in the future could minimize the problems.

In conclusion, the prevalence of neonatal hyperbilirubinemia in this study is $4.08 \%$. The most common causes are physiological causes, followed by neonatal hepatitis, other hemolytic causes, infections, ABO incompatibility, breastfeeding jaundice, sepsis, cephalohematoma, other nonhemolytic causes, drug-induced hepatitis and billiary atresia. The high number of neonatal hyperbilirubinemia cases indicates that neonatal hyperbilirubinemia cases are common and remain as one of the neonatal problems at Dr. Hasan Sadikin General Hospital. Therefore, proper management of neonatal hyperbilirubinemia cases by health care practitioners should be improved. Prevention of severe neonatal hyperbilirubinemia should be implemented by members of the society.

\section{References}

1. Taeusch HW, Ballard RA, Gleason CA, Avery ME. Avery's diseases of the newborn. $9^{\text {th }}$ ed. Philadelphia: Elsevier Saunders; 2012. p. 1129-1148.

2. Brown AK. Kernicterus: past, present, and future. Pediatrics. 2003;4(2):e33-40.

3. Kliegman RM, Stanton BMD, Geme JS, Schor NF, Behrman RE. Nelson textbook of pediatrics: expert consult. $19^{\text {th }}$ ed. Philadelphia: Elsevier Health Sciences; 2011. p. 603-8.

4. Setia S, Villaveces A, Dhillon P, Mueller BA. Neonatal jaundice in Asian, white, and mixed-race infants. Arch Pediatr Adolesc Med. 2002;156(3):276-9.

5. $\mathrm{Du} \mathrm{L}, \mathrm{Ma} \mathrm{X}$. International perspectives: hyperbilirubinemia and kernicterus in neonates in China. Neoreviews. 2012;13(3):e141-4.

6. Maisels MJ. Neonatal jaundice. Pediatr Rev. 2006;27(12):443-54.

7. National Institute for Health and Care Excellence. Neonatal jaundice: NICE clinical guideline 98. Manchester: 
National Institute for Health and Clinical Excellence. 2010 [cited 2013 March 21]: Available from: http://www.nice.org.uk/ nicemedia/live/12986/48678/48678.pdf.

8. Sgro M, Campbell D, Shah V. Incidence and causes of severe neonatal hyperbilirubinemia in Canada. CMAJ. 2006;175(6):587-90.

9. Widiasta A, Reniarti L, Sukadi A. Incidence of neonatal hyperbilirubinemia in low, intermediate-low, and intermediate-high risk group infants. Paediatr Indones. 2010;50(6):351-4.

10. Indriyani SAK, Retayasa IW, Surjono A, Suryantoro P. Percentage birth weight loss and hyperbilirubinemia during the first week of life in term newborns. Paediatr Indones. 2009;49(3):149-54.

11. Tikmani SS, Warraich HJ, Abbasi F, Rizvi A, Darmstadt GL, Zaidi AK. Incidence of neonatal hyperbilirubinemia: a populationbased prospective study in Pakistan. Trop Med Int Health. 2010;15(5):502-7.

12. Moerschel SK, Cianciaruso LB, Tracy LR. A practical approach to neonatal jaundice. Am Fam Physician. 2008;77(9):1255-62.

13. Cloherty JP, Eichenwald EC, Hansen AR,
Stark AR. Manual of neonatal care. 7th ed. Philadelphia: Wolters Kluwer Health; 2012. p. 304-39

14. Lazarus C, Avchen RN. Neonatal hyperbilirubinemia management: a model for change. J Perinatol. 2009;29(Suppl 1):S58-60.

15. Nold JL, Georgieff MK. Infants of diabetic mothers. Pediatr Clin North Am. 2004;51(3):619-37, viii.

16. Gudmundsson S, Henningsson AC, Lindqvist P. Correlation of birth injury with maternal height and birthweight. BJOG. 2005;112(6):764-7.

17. AAP Subcommittee on Neonatal Hyperbilirubinemia. Neonatal jaundice and kernicterus. Pediatrics. 2001;108(3):7635.

18. Maisels MJ. Neonatal hyperbilirubinemia and kernicterus-not gone but sometimes forgotten. Early Hum Dev. 2009;85(11):727-32.

19. Manning D, Todd P, Maxwell M, Platt MJ. Prospective surveillance study of severe hyperbilirubinaemia in the newborn in the UK and Ireland. Arch Dis Child Fetal Neonatal Ed. 2007;92(5):F342-6 